\title{
EMITTANCE AND ENERGY CONTROL IN THE NLC MAIN LINACS*
}

\author{
C. Adolphsen, K.L.F. Bane, K. Kubo, T. Raubenheimer, R.D.Ruth, K.A. Thompson, F.Zimmermann, \\ Stanford Linear Accelerator Center, Stanford University, Stanford, CA 94309 USA
}

\begin{abstract}
We discuss tolerances and correction schemes needed to control single- and multi-bunch emittance in the NLC main linacs. Speci®cations and design of emittance diagnostic stations will be presented. Trajectory correction schemes appropriate to simultaneously controlling the emittance of a multibunch train and the emittance of individual bunches within the train will be discussed. We discuss control of bunch-to-bunch energy spread using a ramped RF pulse generated by phase-modulating the SLED-II input. Tolerances on ions, wake ®elds, quadrupole alignment, and accelerating structure alignment will be given.
\end{abstract}

\section{INTRODUCTION}

The X-band linacs in the NLC will accelerate low emittance electron and positron bunch trains from $10 \mathrm{GeV}$ to $250 \mathrm{GeV}$ in the initial phase of running, and from $10 \mathrm{GeV}$ to $500 \mathrm{GeV}$ after upgrades to the rf system. For initial (upgraded) operation, trains of $90(75)$ bunches with $0.65 \times 10^{10}\left(1.1 \times 10^{10}\right)$ particles per bunch, and a $1.4 \mathrm{~ns}$ spacing between bunches, will be accelerated at a repetition rate of $180(120) \mathrm{Hz}$. The X-band accelerator structures that will provide the high acceleration gradient $(50 \mathrm{MeV} / \mathrm{m}$ unloaded during initial operation and $85 \mathrm{MeV} / \mathrm{m}$ after upgrades), will also heavily load the beam $(25 \%$ by the last bunch) and produce strong long-range and short-range transverse wake®elds when the beams are off-axis. The preservation of the emittance of the beams (nominally $\gamma \epsilon_{x}=3 \times 10^{-6}$ and $\left.\gamma \epsilon_{y}=3 \times 10^{-8}\right)$ and the energy spread $(\sim 0.1 \%)$ will require tight alignment and rf control tolerances. Meeting these tolerances will require that various beam-based corrections schemes be employed during operation. In the following sections, we discuss some of the tolerances and correction schemes after giving a brief description of the linac layout.

\section{LINAC LAYOUT}

The main linac will be basically an array of X-band accelerator structures interleaved with a FODO quadrupole lattice and interspersed with beam diagnostic devices. In order to provide maximum -exibility for using beam-based methods to control beam emittance growth, the accelerator structures and quadrupole magnets (quads) will contain beam position monitors (BPMs) and will be supported on remotely controlled mechanical movers.

The current design for the NLC main linac accelerating structure incorporates both detuning and damping, where the damping is accomplished by coupling all cells in the structure to four parallel manifolds[2][3]. The $Q$ 's of the modes in the lowest dipole passband are lowered to about 1000 , which is suf®cient to control multibunch beam break-up. The manifolds will also

\footnotetext{
*Work supported by the Department of Energy, contract
} DE-AC03-76SF00515. serve as BPMs that provide measures of the beam position relative to the structure axes. The $1.8 \mathrm{~m}$ long accelerator structures will be supported in pairs on a common strongback, and the strongback itself will be supported by movers that have independent horizontal and vertical positioning control at two points along the strongback. The structure movers will attach to a common girder, which itself will attach to the beam line support pedestal via manually adjustable supports. These girder supports will be used to set the initial position and orientation of the structure pairs.

At the beginning of the linacs, a quadrupole magnet will be located after each structure pair. The separation of the quads will increase in two-structure increments along the linacs, from one pair of structures to ${ }^{\circledR}$ ve pairs at the end of the linacs. The magnet lengths will likewise increase, from about $0.1 \mathrm{~m}$ to $1 \mathrm{~m}$. Each quad will contain a stripline BPM in its bore, and each quad/BPM unit will be mounted on a magnet mover, which in turn will be mounted on a manually adjustable support. Together this system will have the same adjustment capability as the structures.

\section{ALIGNMENT AND TRAJECTORY CORRECTION}

Misalignments of the quadrupole magnet centers about the nominal linac axis will produce dispersion and hence beam emittance growth because of the non-zero energy spread. Methods that are generally used to align the quads have accuracies that depend on the distance scale of the alignment, so it is useful to characterize the alignment tolerance in terms of the wavelength of the misalignments. In computing these tolerances, we assume that the quad offsets are sinusoidal with wavelength $\lambda$, and that bunches are steered to zero in the BPMs, which themselves are centered in the quads. Figure 1 shows the tolerance on the misalignment amplitude for a 3\% vertical emittance growth as a function of $\lambda$. The rapid rise in the tolerance above $160 \mathrm{~m}$ occurs since $\lambda$ becomes larger than the longest betatron wavelength in the linac, so the dispersion averages out.

The initial placement of the quads and structures will be done with conventional surveying techniques. Most likely, triangulation and leveling methods will be used in combination with Global Positioning System (GPS) data from satellites. The longrange alignment accuracy will be within the dispersion related tolerances shown in Figure 1. However, at wavelengths less than a few hundred meters, the accuracy will level off to values that approach 100 microns on a $20 \mathrm{~m}$ scale. At these wavelengths, beam-based methods will be used to control the quad alignment (these methods tend to be sensitive to systematic errors at longer wavelengths and hence it is better to rely on placement accuracy to achieve these tolerances). The quad alignment corrections computed by these methods are generally highly correlated, so one does not usually refer to the absolute level of quad align- 
ment, but instead to the residual dispersion remaining after alignment, which is a function of the BPM resolution and the alignment accuracy of the BPMs relative to the quad centers.

The quickest and simplest beam-based quad alignment algorithm is one that uses only the beam trajectory data taken with the nominal linac lattice. Having readings from N BPMs in N quads allows one to determine $\mathrm{N}-1$ quad offsets, with the alignment end-points being the beam position in the $®$ rst quad and the BPM zero of the last quad: the outgoing beam angle is unconstrained. One would align N-1 quads at a time, one group after the next, using the magnet movers to control the quad positions to the micron level. Values of $\mathrm{N}$ that would be practical range from 50 to 100 , or $1 / 14$ to $1 / 7$ of the total number of quads in each linac.

Although this method would only require that the quad BPMs have resolutions of a few microns, it also requires that their mechanical plus electronic offsets relative to the quad magnetic centers be known to this same level. These offsets can be computed in a beam-based manner as well, but this requires changing the linac quad settings and would slow down the quad alignment process and disrupt colliding beam operation. To minimize the impact of such measurements, the BPM system will be designed to either insure that any drifts in the offsets after measurement will be accurately monitored, or that the changes will not be signi®cant on at least a 24 hour time scale so that at most one measurement a day would be needed. Achieving stable BPM offsets will be especially important if the quad alignment algorithm needs to be implemented as a feedback loop in order to keep up with the effect of ground motion changes on short (hourly) time scales.

Another potentially large contributor to beam emittance growth in the NLC linacs is the transverse wake®eld that is generated as the bunches travel off-axis through the structures. The wake®eld degrades both the beam emittance (i.e., it generates bunch-to-bunch orbit variations) and the bunch emittances (i.e., it generates differential kicks along the longitudinal bunch pro(les). Although the structure detuning and damping will significantly suppress the strength of the long-range wake®eld, and BNS damping will effectively cancel the effect of the short-range wake®eld on betatron motion, the alignment tolerances for the structures are still tight.

In computing theses tolerances, we suppose that the quads and BPMs are perfectly aligned, and that the net wake@eld kick to the beam is removed locally by steering the beam centroid to zero in the BPMs. As in the dispersion case, we consider misalignments on different length scales, although in this case we assume piecewise misalignments as opposed to sinusoidal, where the piece lengths vary from sub-structure sizes to multi-structure groups [4]. Using conventional optical alignment techniques, it would be extremely dif®cult to achieve the required tolerances (of order 10 microns) at scales greater than a few structure lengths. Instead, the structure mover system will be used to align the two structures as a whole relative to the beam trajectory based on the dipole mode signals from the structure damping manifolds. Signals from two modes near the ends of the structure will be selected with $®$ Iters and measured so that both the position and orientation of the structure relative to the beam can be determined. The average of the measurements from the two structures will be

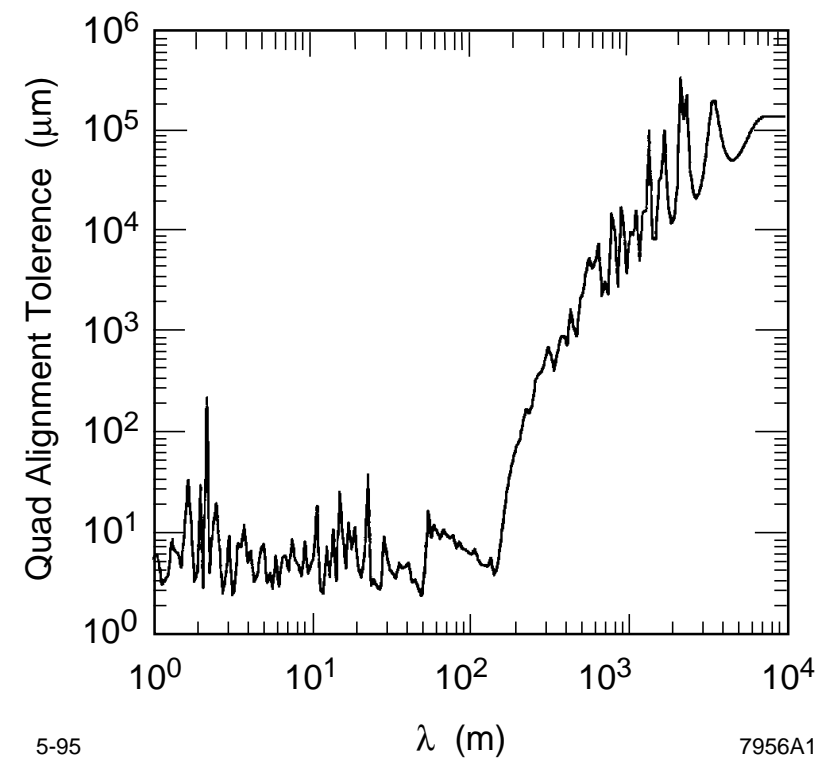

Figure 1. Misalignment tolerance for a $3 \%$ vertical emittance growth as a function of misalignment scale $\lambda$.

used to remotely adjust the mover positions at the micron level to better center the pair about the beam trajectory. This procedure will be iterated with the quad alignment algorithm if signi®cant orbit changes occur.

The difference in the measurements from each structure pair will be used to monitor the relative structure alignment on the strongback. This alignment and the internal alignment of each of the two structures will be established prior to installation by optical means. There will be six supports on each structure that can be adjusted to meet the required tolerances; the tolerances on shorter length scales will be achieved by precision assembly of the cells prior to brazing. Adjustments to the six supports on each structure will still be possible in-situ, although not easily given the precision required.

\section{ION EFFECTS}

In the NLC linacs, ions are created by collisional ionization of the residual gas. Light ions, such as hydrogen, are overfocused and lost between bunches, while, in the ®rst part of the linac, heavier ions are trapped within a bunch train. Trapped ions affect the beam dynamics in three different ways. First, the additional focusing of the trailing bunches due to the ions will lead to an increased $₫$ lamentation which is insigni $\AA$ cant only for a vacuum pressure below $10^{-8}$ Torr [5]. Second, the ions cause a nonlinear octupole-like coupling of horizontal and vertical betatron motion, whose effect is greatly reduced when the horizontal and vertical phase advances are separated by about 5\% [5]. Third, the coupled motion of beam and ions may result in a fast transverse multi-bunch instability of the electron bunch train in the linacs. If the pressure is $10^{-8}$ torr, the expected instability rise time at the start of the main linac is about $160 \mathrm{~ns}$ [6], assuming 90 bunches of $6.6 \cdot 10^{9}$ particles each and carbon monoxide ions (CO). The beam-ion instability disappears when the ions are no longer trapped within the train. The distance at which this happens depends on the beam current and on the ion mass. As an 
example, for a bunch train of 90 bunches and $6.6 \cdot 10^{9}$ particles per bunch, $\mathrm{CO}$ ions are trapped up to a beam energy of about 38 $\mathrm{GeV}$, which corresponds to a distance of about $800 \mathrm{~m}$ in the main linac. For an average $\mathrm{CO}$ pressure of $2 \cdot 10^{-8}$ torr and an initial bunch-to-bunch offset of $0.01 \sigma_{y}$ the expected total dilution of the vertical emittance due to the beam-ion instability is then about $4 \%$. At $5 \cdot 10^{-8}$ torr the dilution would exceed $100 \%$. For higher beam intensities fewer ions are trapped over a shorter distance, and the pressure tolerance is looser.

\section{MULTIBUNCH ENERGY CONTROL}

The method of multibunch energy compensation is to linearly ramp the input RF pulse during one ®lling time just prior to injection of the beam [7]. Without such compensation, there would be a drop of energy of about $25 \%$ from the head of a train to the tail, due to beam loading of the accelerating mode. The bandwidth of the present NLC $®$ nal focus design is $\pm 0.7 \%$ [8]. Thus, the multibunch energy spread and the variation in the average energy of the beam must both be controlled to a few tenths of a percent. The simple linear ramp gives quite good compensation, but the RF pulse could be further corrected to improve the compensation (and maintain it via feedback as conditions vary).

Parameters used in the simulations are as follows: RF frequency, $f_{r f}=11.424 \mathrm{GHz}$, section length $=1.8 \mathrm{~m}$, attenuation $\tau=0.505$, fundamental mode $Q=7107$, fundamental mode loss factor $\kappa_{1}=203.75 \mathrm{~V} / \mathrm{pC}$, ®lling time, $T_{f}=100 \mathrm{~ns}$, bunch spacing $=16 \lambda_{r f f} \approx 42 \mathrm{~cm}$, bunch charge $=1 \times 10^{10}$. We model the linac as made up of CG sections, with $2 \pi / 3$ phase advance per cell.

The bunches must be placed ahead of the RF crest (by about $13^{\circ}$ for the parameters used here), to compensate the intrabunch energy spread. When the energy spread is optimized, there is a residual rms fractional energy spread (including both intrabunch and bunch-to-bunch spread) of about $0.2 \%$.

We examined the sensitivity of the rms energy spread and the mean energy of the multibunch beam to bunch length, bunch charge variations, and ripple of the incoming RF pulse. The optimum energy compensation is not very sensitive to bunch length. A $20 \%$ change in bunch length away from the nominal value of $100 \mu$ m produces an additional rms energy spread of about $0.1 \%$.

The compensation is quite sensitive to systematic changes in bunch charge, i.e., changes that are similar for all bunches in the train. Changing all the bunch charges by $2 \%$ from their optimum value increases the rms fractional energy spread from $0.2 \%$ to $0.3 \%$. Changing all the bunch charges by $0.3 \%$ from their optimum value produces a shift of about $0.1 \%$ in the centroid energy of the beam.

The sensitivity to RF phase and amplitude ripple was studied as a function of time scale and amplitude of the ripple. The tightest tolerances occur for ripple that has large variations on a time scale comparable to the $100 \mathrm{nsec}{ }^{\circledR}$ lling time, i.e. if the ripple is taken to be sinusoidal, the tightest tolerances occur for sinusoids with periods of 200 to $400 \mathrm{nsec}$. For shorter time scales, the ripple partly averages out over a ${ }^{\circledR}$ lling time, loosening the tolerances. For longer time scales, the rms energy spread tolerances loosen somewhat, while the centroid energy tolerances remain about the same. The tolerances also depends on whether the ripple is similar in all accelerating sections $Đ$ if it is ra ndom from section to section, the tolerances are of course looser. The most pessimistic estimate, assuming a tolerance of $0.1 \%$ increment to the rms energy spread, is $\pm 0.5^{\circ}$ for the phase ripple and $\pm 0.3 \%$ for the ®eld-amplitude ripple. The most pessimistic estimate, assuming a tolerance of $0.1 \%$ energy centroid shift, is $\pm 0.3^{\circ}$ for the phase ripple and $\pm 0.3 \%$ for the Beld-amplitude ripple.

\section{References}

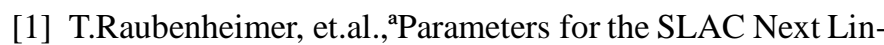
ear Collider ${ }^{\circ}$, this conference.

[2] N.Kroll, et.al., ${ }^{a}$ Manifold Damping of the NLC Detuned Accelerating Structure ${ }^{\circ}$, 6th Workshop on Advanced Accelerator Concepts,, Lake Geneva, WI, 12-18 June 1994; SLACPUB-6660.

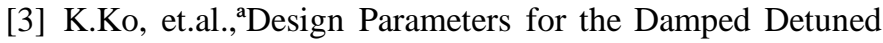
Accelerating Structure ${ }^{\circ}$, this conference.

[4] K.Kubo, et.al., 'A Alignment Tolerances of A ccelerating Structures and Correction Schemes for Future Linear Colliders ${ }^{\circ}$, this conference.

[5] T. Raubenheimer and P. Chen, ${ }^{\mathrm{a}}$ Ions in the Linacs of Future Linear Colliders ${ }^{\circ}$, presented at LINAC 92, Ottawa,(1992); SLAC-PUB-5893.

[6] T.Raubenheimer and F.Zimmermann, ${ }^{\mathrm{a}} \mathrm{A}$ Fast Beam-Ion Instability ${ }^{\circ}$, this conference.

[7] K.A. Thompson and R.D. Ruth, Proceedings of the 1993 Particle Accelerator Conference, May 17-20, 1993, Washington, D.C.; SLAC-PUB-6154.

[8] F.Zimmermann et.al., aA Final Focus System for the Next Linear Collider ${ }^{\circ}$, this conference. 